\title{
Using a parallel micro-cultivation system (micro-Matrix) as a process development tool for cell culture applications
}

\author{
Vincent Wiegmann ${ }^{1}, \cdot$ Cristina Bernal Martinez ${ }^{2}, \cdot$ Frank Baganz ${ }^{1 *}$ \\ *corresponding author \\ e-mail: f.baganz@ucl.ac.uk \\ ${ }^{1}$ The Advanced Centre for Biochemical Engineering, Department of Biochemical Engineering, University \\ College London, Gower Street, London, WC1E 6BT \\ ${ }^{2}$ Applikon-Biotechnology BV. Heertjeslaan 2, 2629JG. Delft, The Netherlands
}

\section{Keywords}

Miniature shaken bioreactor $\cdot$ micro-Matrix $\cdot$ Fed batch $\cdot$ GS-CHO cells $\cdot$ Scale down 


\section{Abstract}

Micro-bioreactors appear frequently in today's biotechnology industry as screening and process development tools for cell culture applications. The micro-bioreactor's small volume allows for a high throughput and when compared to other small scale systems, such as microtitre plates, its measurement and control capabilities offer a much a better insight into the bioprocess. Applikon's micro-Matrix is one of the micro-bioreactors that are commercially available today. The microMatrix system consists of shaken disposable 24 deep square well plates in which each well is controlled individually for $\mathrm{pH}$, dissolved oxygen (DO), and temperature. Additionally, a feeding module supports automated additions of liquid to each well. This chapter describes how the microMatrix can be used for fed-batch cultivations of Chinese Hamster Ovary (CHO) cells.

\section{Introduction}

Throughput is a decisive factor in driving the development of upstream processes forward. Conventional stirred tank bioreactors (STRs) are unpractical for extensive optimisation studies due to their slow turnover and high spatial demand. Typically, microwell systems are used for early stage development such as clone screening, before employing shake flask cultures for media development or to design feeding strategies [1]. Although both systems allow for moderate to high throughput, a lack of monitoring and control curtails the information density in comparison to STRs. Microbioreactors bridge this gap between bioreactors and microwell systems by offering a fully-controlled culture environment on a small scale. This allows for the quick accumulation of process knowledge early on in the development timeline, which can speed up development whilst keeping mediarelated costs to a minimum [2].

Several developments were instrumental in the recent advancement and success of microbioreactors. Monitoring and control of $\mathrm{pH}$ and $\mathrm{DO}$ at the small scale has been enabled by the availability of affordable, small, non-invasive sensors. Additionally, routine offline analyses such as antibody titre, viable cell concentration, and metabolites can now be performed at submillilitre volumes, which allows to fully utilise the high degree of parallelisation. Micro-bioreactors also frequently incorporate single-use materials, which substantially reduces the turnover time when compared to glass or stainless steel vessels and allow runs to be scheduled in rapid succession [3]. In general, micro-bioreactors can be divided into either shaken or stirred systems. The design of stirred systems (e.g. ambr15, bioREACTOR) follow similar operational principles to larger scale vessels, where a stirrer is inserted into the liquid from above and a sparger provides aeration. 
Shaken systems (e.g. micro-Matrix, BioLector), on the other hand, rely on overhead aeration [4], [5]. However, to date only the ambr15 and the micro-Matrix have been documented in the literature for cell culture applications [2], [5]-[8].

Micro-bioreactors exist with various degrees of automation. In many cases, liquid-handling robots can be integrated with the micro-bioreactor system (e.g. RoboLector, ambr15) to facilitate routine interactions such as sampling or the addition of feed medium [6], [7], [9]. However, alternative approaches have also been explored. For example, Applikon's micro-Matrix enables automated liquid additions by connecting each culture compartment to individual liquid lines, allowing for nearcontinuous feeding without running the risk of overloading the schedule of a pipetting robot [10]. An overview of the today's commercially available micro-bioreactors options is given in Table 1.

Applikon's micro-Matrix (Applikon, Delft, The Netherlands) is a platform that holds 24 individual micro-bioreactors based on a 24 deep-well cassette with 4 independent gas inlets for overhead aeration and one liquid feed line. The inbuilt orbital shaker operates at shaking speeds between $0-400 \mathrm{rpm}$ at an orbital throw of $25 \mathrm{~mm}$. The cassette is placed in a temperature-controlled chamber at a defined setpoint. Additionally, the temperature can be controlled individually for each well, with a maximum difference of $1^{\circ} \mathrm{C}$ between adjacent wells [11], [12]. For cell culture processes, the DO is typically controlled with nitrogen and air. As evaporation is of particular concern in the small scale, pure oxygen can be used instead of air to further decrease gas flow rates and by extension, minimise evaporation. Control of the $\mathrm{pH}$ is achieved through overlay with carbon dioxide. Furthermore, the liquid feed line can be used for automated additions of base. The parameters DO, $\mathrm{pH}$, and temperature are maintained through PID control loops that can be customised for each well individually.

The protocol outlined here describes how the micro-Matrix can be used for fed-batch cultivations of $\mathrm{CHO}$ cells, where the automated feeding module achieves the feed additions.

[Table 1 about here] 


\section{Materials}

2.1 Cell culture

1. CHO Cell line (See Note 1)

2. Chemically defined basal and feed medium (See Note 1)

3. Bicarbonate buffer: $250 \mathrm{mM} \mathrm{Na} 2 \mathrm{CO} 3$ and $250 \mathrm{mM} \mathrm{NaHCO} 3$

2.2 Preculture

1. Shake flask (See Note 2)

2. Certomat MO II shaker with $25 \mathrm{~mm}$ orbit (Sartorius)

3. MCO-18M 02/CO2 Incubator (Sanyo)

\subsection{Micro-Matrix cultures}

1. micro-Matrix micro-bioreactor system (Applikon-Biotechnology)

2. Disposable micro-Matrix cassette (Applikon-Biotechnology) with pre-calibrated optical sensors for $\mathrm{pH}$ and $\mathrm{DO}$

3. Disposable micro-Matrix filter bars (Applikon-Biotechnology)

2.5 Cell Count

1. Vi-CELL XR (Beckman Coulter)

2.6 Analysis of nutrient and metabolite concentrations

1. Bioprofile Flex (Nova Biomedical)

2.5 Quantification of antibody titre

1. High-performance liquid chromatography (HPLC) (See Note 3)

2. Protein $\mathrm{G}$ column (See Note 3 )

3. Phosphate buffer at $\mathrm{pH} 7$ for loading and washing step

4. Glycine buffer at $\mathrm{pH} 2.8$ for elution 


\section{Methods}

\subsection{Preculture}

1. Remove a vial from the working cell bank stored in liquid nitrogen and thaw in a water bath at $37^{\circ} \mathrm{C}$ (See Note 21). Transfer vial content into a falcon tube and dilute 10 times with fresh medium. Centrifuge at $1000 \mathrm{~g}$ for $5 \mathrm{~min}$ and discard the supernatant. Resuspend the cell pellet in $\mathrm{CD}-\mathrm{CHO}$ with $25 \mu \mathrm{M}$ MSX and transfer into a shake flask. Adjust the cell concentration to $0.3 \mathrm{x}$ $10^{6}$ cells $\mathrm{mL}^{-1}$.

2. Expand the cells on a shaker in a humidified incubator at $37^{\circ} \mathrm{C}, 5 \% \mathrm{CO}_{2}, 95 \%$ air, and $160 \mathrm{rpm}$. Passage cells regularly and observe growth for at least 7 days prior to starting the experiment (See Note 5).

\subsection{Initial setup}

1. Turn on the system via the switch on the right-hand side of the machine.

2. Connect gas supply of oxygen, nitrogen, and carbon dioxide to three of the gas inlet connectors at the back of the device. Using regulators, the inlet pressure should be set between $2-6$ bar (See Note 1 and 15).

3. Connect the Ethernet RJ45 connector to a PC with the control software.

\subsection{Calibrating the feeding module}

The micro valves of the Liquid Addition Top Plate need to be calibrated for every liquid individually. This procedure has to be performed prior to autoclaving the module (See Note 12).

1. Fill the Liquid Addition Feed Bottle (LAFB) with the liquid that the micro valves need to be calibrated for and connect the LAFB to the compressed gas outlet inside the micro-Matrix cabinet (See Note 13).

2. Connect the Liquid Addition Top Plate to the LAFB, place it over a waste container, and connect the control box to the delivery module.

3. In the HMI software click 'Actuator and PID setup' -> 'Liquids Library'. Create a new liquid, insert a name, and select the pulse time (See Note 14). Select the number of pulses and click 'Start calibration' to start the delivery of liquid. Run the calibration until all lines have been primed.

4. Now, place the feeding module on a rack with pre-weighed centrifuge tubes and repeat step 3. 
5. After all pulses have been delivered, weigh the centrifuge tubes and calculate the delivered volume by taking the density of the liquid into account. Dividing with the number of pulses, the volume per pulse can be calculated and must then be inserted in the 'Pulse volume' section.

6. Save the values by clicking the green tick.

7. Before autoclaving the feeding module, it needs to be cleaned as outlined in Section 3.11. to avoid feed residues blocking the tubing or micro valves

\subsection{Calibration and Re-calibration of $\mathrm{pH}$ sensors}

1. After defining the Recipe, the calibration values can be entered by clicking 'Calibrate the microMatrix' on the main screen and either scanning the QR code on the cassette, entering the batch number of the cassette, or manually entering the calibration values.

2. Before starting the experiment, the $\mathrm{pH}$ offsets should be determined by aseptically filling each well of the cassette that will be used for the experiment with at least $2 \mathrm{~mL}$ of medium and mounting the assembled cassette onto the micro-Matrix without connecting the gas lines (See Note 7).

3. Initiate $\mathrm{pH}$ measurements by starting the experiment without agitation, $\mathrm{DO}, \mathrm{pH}$, or temperature control. Let the probes equilibrate for 1-2 hours or until the measurements reach a plateau (See Note 6).

4. Aseptically remove an appropriate amount of the medium from each to perform offline $\mathrm{pH}$ measurements and note the offline value for each well.

5. Continue the run with the residual medium still in the wells.

6. While the device is running, click 'Calibrate the micro-Matrix', select the ' $\mathrm{pH}$ measurement calibration' tab, and tick the box 'Upload offline $\mathrm{pH}$ values'. Enter the offline values for each well and click OK (See Note 8).

\subsection{Inoculation}

1. Perform a cell count of the preculture. Based on the viable cell density, prepare the inoculum with a final cell count of $0.3 \times 10^{6}$ viable cells $\mathrm{mL}^{-1}$ by diluting with fresh basal medium (See Note 1 and 20).

2. In the laminar flow cabinet, remove the cassette carefully from the packaging. The cassette has a plastic cover, which has to be removed without compromising the sterility of the plate (See Note 20). 
3. Aseptically take up the well-mixed inoculum (shake before use) using a pipette and dispense the required volume into each well of the cassette (See Note 19).

4. Aseptically fill the LAFB with approximately $200 \mathrm{~mL}$ of the feed medium (this can also be done at a later stage of the process to avoid degradation of the medium).

5. Now, the lines of the Liquid Addition Top Plate need to be primed. To do so, pressurise the bottle using a syringe and place the Feeding Module over a sterile container (e.g. the plastic lid of the cassette). Connect the priming module and turn the switch to the 'On' position. All valves will now successively open and dispense some of the liquid. After two cycles, the priming module can be dismounted.

6. Then, place the Liquid Addition Top Plate onto the cassette and use the two metal clamps to hold it in place. Note that the cover only fits in one orientation. Row D should align with the 'Front' written on the Liquid Feeding Module.

7. Aseptically remove the Filter Bars from the packaging and fit them onto the Liquid Addition Top Plate. Small plastic fittings indicate the correct orientation of the Filter Bars. (Note: Force may be necessary to slot the gas bars in place).

8. Mount the assembled cassette onto the micro-Matrix, use the transport clamps to hold the cassette in place, and attach the Gas Bars to the Filter Bars. Finally, fasten the Control Box to the Liquid Addition Top Plate (See Note 17).

\subsection{Actuator selection}

1. Click 'Actuator and PID setup' in the main screen to open the configuration menu as shown in Figure 1.

2. Select '24 wells cassette' under 'Cassette layout'.

3. Select the appropriate liquid from the drop-down menu (see 3.3 for Calibration of the Feeding Module) under 'Liquids configuration'.

4. Highlight one or more wells in the 'Reactor selection' field. Any changes made in the following fields will be valid for all selected wells.

5. Select a suitable PID setup from the drop-down menu under 'Control configuration'. The PID setting can be customised for each well individually if necessary (See Note 9, 10, 11, and 16).

6. Define a cut-off volume for the liquid delivery.

7. In the 'Actuator configuration' field, tick the boxes of the gases that will be used in the experiment and assign them to their corresponding actions via drag and drop (See Note 15).

8. Levels can be populated if a cascade control chain is desired.

9. Click OK to save and return to the main screen. 
[Figure 1 about here]

\subsection{Recipe setup}

The micro-Matrix software allows for a range of process conditions and feeding regimes. The following instructions describe a fed-batch protocol in which feeding commences after 72 hours of cultivation with a constant rate of $100 \mathrm{~nL} \mathrm{~min}^{-1}$ (See Note 23).

1. Click 'Recipe' in the main screen to open the configuration menu as shown in Figure 2.

2. Define the desired setpoints for $\mathrm{DO}, \mathrm{Temperature,} \mathrm{pH}$, and shaking speed.

3. Create a second process period by clicking 'Add new period' in the top left corner and define the duration of Period 1 to be 72 hours (hhhh:mm).

4. Check that the setpoints are identical in both periods.

5. In the 'Direct control' tab, define the flow rate of feed medium. In Period 1 the flow rate is set to be $0 \mathrm{~nL} \mathrm{~min}-1$ and in Period 2 it is set to be $100 \mathrm{~nL} \mathrm{~min}$. In the 'Alarm' tab, upper and lower bounds of the process parameters can be defined. If one or more of the measured process parameters fall outside these specifications, the green light on the device will turn red.

[Figure 2 about here]

\subsection{Sampling}

Sampling can either be done on a sacrificial basis or on an individual basis. In case of the sacrificial sampling, the entire volume of one well is removed, whereas in the case of the individual sampling only a portion of the working volume is extracted. In this protocol, only individual sampling is considered.

1. Unclamp the plate, take it from the shaker, and weigh it to determine the plate-wide liquid loss due to evaporation.

2. Transfer the assembled cassette and feeding bottle to the laminar flow cabinet. Carefully remove the top plate from the cassette. The gravimetrically determined liquid loss can now be compensated with $\mathrm{DI} \mathrm{H}_{2} \mathrm{O}$, before the required sample volume is extracted from each well and transferred to labelled Eppendorf tubes (See Note 18).

3. Optionally, bicarbonate buffer can be added for $\mathrm{pH}$ control. 
4. Assemble and weigh the cassette before mounting it on the OTM (see Section 3.5).

5. Use part of the cell suspension to determine the cell concentration (at appropriate dilution with 1XPBS - take the limited volume into consideration) (See Note 22).

6. Centrifuge the remaining suspension at $16.1 \mathrm{k}$ rcf for $5 \mathrm{~min}$ to pellet the cells.

7. The supernatant can be stored at $-18^{\circ} \mathrm{C}$ for later analysis.

\subsection{Ending an experiment and export of data}

1. Terminate the running experiment by clicking 'Stop Experiment'.

2. Now select between micro-Matrix data file, Excel file, or Text file format.

3. Click 'Export' to define the location and name of the exported data file (See Note 24 and 25).

\subsection{Cleaning}

Although most of the materials such as Filter Bars and Cassette are disposable, particularly the Liquid Feeding Module needs to undergo a cleaning procedure after use to avoid blockage of tubing and micro valves.

1. Remove the Liquid Addition Top Plate from the used cassette, position it over a container, and replace the residual feed medium in the LAFB with hot distilled water.

2. Pressurise the bottle using a syringe or compressed air, connect the priming module, and turn the switch to the 'On' position. All valves will now successively open and dispense some of the liquid. Rinse the lines for at least 2 min.

3. Now perform a backflush by immersing the Top Plate in hot distilled water and creating a vacuum in the LAFB using a syringe. Again, turn the switch of the priming module to the 'On' position and continue the backflush for at least $2 \mathrm{~min}$.

4. Before storing the Liquid Feeding Module, perform an air flush by repeating Step 2 with an empty LAFB.

\section{Notes}

1. The micro-Matrix was used by the authors to culture a GS-CHO cell line (Lonza) expressing a monoclonal antibody. CD-CHO (Life-Technologies) was used as basal medium and Efficient Feed B (Life-Technologies) as feed medium. Methionine sulfoximine (MSX) (Sigma-Aldrich) was used in routine passages to amplify antibody expression. 
2. Routine passages were performed in $250 \mathrm{~mL}$ shake flask with vent cap (Corning Life Sciences).

3. Titre analysis was performed with an Agilent 1200 (Agilent Technologies) together with a 1 mL HiTrap Protein G HP column (GE Healthcare).

4. A liquid leak detector can be used to check gas connections. Gas leaks can lead to an unexpected depletion of the gas supply and should be avoided.

5. Setting the working volume of shake flasks to $20 \%$ of their nominal volume ensures a reasonable surface to volume ratio in terms of aeration. A higher working volume may lead to limitations in the oxygen transfer, while lower fill volumes may induce out-of-phase motion of the liquid.

6. As the $\mathrm{pH}$ readings are affected by temperature, online and offline readings for the $\mathrm{pH}$ recalibration should be done at room temperature. By entering ' $X$ ' as setpoint, the temperature control can be disabled. Similarly, the gas bars should not be connected to avoid inadvertent $\mathrm{pH}$ changes.

7. Instead of medium, sterile 1 XPBS can be used to determine the $\mathrm{pH}$ offset as it provides more stable $\mathrm{pH}$ values. However, the $1 \mathrm{xPBS}$ will have to be removed prior to starting the experiment.

8. Recalibration of the $\mathrm{pH}$ cannot be transferred between experiments. Therefore, recalibration and the actual experiment have to be run within the same project.

9. The maximum gas flow rate can be adjusted by changing the value for 'Volume per pulse' under 'Configuration' -> 'Device Settings' -> 'Gas'. Lower gas flow rates can help to reduce evaporation.

10. The PID settings can be adjusted for each individual well under 'Actuator and PID setup' -> 'Control configuration'. Often, accurate control can be achieved with the Proportional Gain alone. If after adjustment of the Proportional Gain a continuous offset persists, a small Integral Gain can be added while reducing the Proportional Gain by $10 \%$. In some cases, Derivative Gain can be useful to reduce oscillation. However, for the control needs of our cell culture processes, control configurations without Derivative Gain suffice.

11. To avoid confusion, each gas inlet can be given a name under 'Configuration' -> 'Device Settings' -> 'Gas'.

12. Autoclaving cycles may affect the dosing rate of the micro valves. It is recommended to recalibrate the liquid feeding module after 5 autoclaving cycles.

13. Any liquid that will be delivered by the feeding module should be $0.45 \mu \mathrm{m}$ filtered before use to increase the lifetime of the micro-valves. 
14. The pulse time defines the quantity of liquid that is delivered with each pulse. The shorter the pulse time, the smaller the doses that the system is capable of delivering.

15. Blends of gasses can be used as needed to accommodate alternative control strategies or process conditions. However, for most standard application pure gases can be used. In cell culture applications compressed air or oxygen are typically used for up-control of the DO, while nitrogen is used for down-control of the DO. Down-control of the $\mathrm{pH}$ is achieved with carbon dioxide, while bicarbonate buffer or base is added for up-control of the $\mathrm{pH}$.

16. If the feeding module is not used for upwards $\mathrm{pH}$ control, the buffer can be added manually. By roughly fixing the molar quantities of buffer to the molar quantities of produced lactate in the medium, we achieve steady $\mathrm{pH}$ control without occupying the feed line with buffer.

17. The LAFB is placed inside the micro-Matrix cabinet by default. However, the cabinet is usually kept at elevated temperatures, which can accelerate degradation of the feed medium. By using a longer piece of gas tubing, the LAFB can be routed outside the cabinet and placed inside a Styrofoam box filled with cooling pads. By replacing the cooling pads every 24 hours, the temperature inside the box can be kept below $5^{\circ} \mathrm{C}$.

18. Multichannel pipettes with adjustable spacer (e.g. Rainin) can facilitate interactions with the microtitre plate such as inoculation, sampling, and feeding.

19. A repeater pipette (e.g. Rainin or Eppendorf) can be helpful to dispense volumes that are otherwise would have to be handled with serological pipettes (e.g. when inoculating the micro-Matrix cassette with $>1 \mathrm{~mL}$ ).

20. Instead of mixing fresh medium and preculture inside the wells, a cell suspension with the final cell concentration should be prepared in a separate sterile vessel (e.g. shake flask), which is then used to fill the wells of the respective cell culture system. This procedure guarantees consistent seeding density.

21. The high DMSO content in the freezing medium causes the viability to decrease rapidly at room temperature. Aim to minimise the time between thawing and dilution in fresh medium.

22. High dilutions with PBS can affect the cell size. If the average cell size is an important parameter, dilutions should be kept as low as possible.

23. Due to the large sample volumes relative to the working volume in the micro-Matrix, feed volumes should be adjusted to avoid overfeeding compared to larger scale cultures. Instead of fixing the feed volume to the initial working volume, it can be helpful to feed based on the current working volume to match feeding strategies between scales more effectively. 
24. To avoid excessive file sizes, the interval between each data point can be increased when exporting the data as Excel files (downsampling).

25. Exported Excel files contain diagrams for all logged data (pH, DO, Temperature, Liquid Delivery, and Gas Delivery). The micro-Matrix files can be opened anytime using the HMI software and allow for review of the logged data within the software interface. The Text file contains the raw values of the logged data.

\section{References}

[1] Doig S, Baganz F, Lye G (2006) High throughput screening and process optimisation. Basic Biotechnol., 3rd ed.

[2] Kelly W, Veigne S, Li X, Subramanian SS, Huang Z, Schaefer E (2018) Optimizing performance of semi-continuous cell culture in an ambr $15^{\mathrm{TM}}$ microbioreactor using dynamic flux balance modeling. Biotechnol Prog 34,2: 420-431

[3] Bareither R, Pollard D (2011) A review of advanced small-scale parallel bioreactor technology for accelerated process development: Current state and future need. Biotechnol Prog 27,1: 2-14

[4] Hemmerich J, Noack S, Wiechert W, Oldiges M (2018) Microbioreactor Systems for Accelerated Bioprocess Development. Biotechnol J, 13,4: 1700141

[5] Wiegmann V, Martinez CB, Baganz F (2018) A simple method to determine evaporation and compensate for liquid losses in small-scale cell culture systems. Biotechnol Lett 40,7: 10291036.

[6] Hsu WT, Aulakh RPS, Traul DL, Yuk IH (2012) Advanced microscale bioreactor system: a representative scale-down model for bench-top bioreactors. Cytotechnol 64,6: 667-78

[7] Nienow AW, Rielly CD, Brosnan K, Bargh N, Lee K, Coopman K, Hewitt CJ (2013) The physical characterisation of a microscale parallel bioreactor platform with an industrial $\mathrm{CHO}$ cell line expressing an IgG4. Biochem Eng J 76: 25-36

[8] Moses S, Manahan M, Ambrogelly A, Ling WLW (2912) Assessment of AMBRTM as a model for high-throughput cell culture process development strategy. Adv Biosci Biotechnol 3,7: 918-927

[9] Chen A, Chitta R, Chang D, Amanullah A (2009) Twenty-four well plate miniature bioreactor 
system as a scale-down model for cell culture process development. Biotechnol Bioeng 102, 1: $148-160$

[10] Applikon, "Brochure: micro-Matrix 24 bioreactors in a convenient microtiter forma," 2016. [Online]. Available: http://www.applikon-biotechnology.us/images/download/micromatrix/micro-Matrix-leaflet.pdf. [Accessed: 30-Jan-2017].

[11] Lattermann C, Büchs J (2015) Microscale and miniscale fermentation and screening. Curr Opin Biotechnol 35: 1-6

[12] DePalma A (2014) Single-Use Bioreactors Dare to Scale. Gen Eng Biotechnol News 34,14:24, $26-27$ 
Using a parallel micro-cultivation system as process development tool

\section{Table captions}

Table 1: Comparison of currently available commercial micro-bioreactor options.

\section{Figure captions}

Figure 1: Actuator Setup in the micro-Matrix HMI

Figure 2: Setup of the experimental conditions in the micro-Matrix HMI. (a) Dissolved oxygen $\left(\mathrm{dO}_{2}\right)$, $\mathrm{pH}$, temperature and shaking speed. (b) Liquid additions 
Table 2: Comparison of currently available commercial micro-bioreactor options.

\begin{tabular}{|c|c|c|c|c|c|c|}
\hline Micro-bioreactor & Agitation & Aeration & $\begin{array}{l}\text { Working } \\
\text { Volume }\end{array}$ & Vessel Format & Special Features & Vendor \\
\hline ambr 15 & Stirred & Sparger & $10-15 \mathrm{~mL}$ & $\begin{array}{l}\text { Disposable micro- } \\
\text { bioreactor vessels }\end{array}$ & $\begin{array}{l}\text { Automated liquid } \\
\text { handling }\end{array}$ & Sartorius-Stedim \\
\hline BioLector ${ }^{\ddagger}$ & Shaken & Overlay & $0.8-2.4 \mathrm{~mL}$ & 48 FlowerPlate & $\begin{array}{c}\text { Can be combined } \\
\text { with liquid } \\
\text { handler }\end{array}$ & $\mathrm{m} 2 \mathrm{p}$-labs \\
\hline bioREACTOR ${ }^{\ddagger}$ & Stirred & Passive & $8-15 \mathrm{~mL}$ & Disposable PS vessels & $\begin{array}{c}\text { Can be combined } \\
\text { with liquid } \\
\text { handler }\end{array}$ & $2 \mathrm{mag}$ \\
\hline micro-Matrix & Shaken & Overlay & $1-5 \mathrm{~mL}$ & $\begin{array}{c}24 \text { Deep Square Well } \\
\text { Plate }\end{array}$ & $\begin{array}{c}\text { Automated } \\
\text { feeding module }\end{array}$ & $\begin{array}{c}\text { Applikon } \\
\text { Biotechnology }\end{array}$ \\
\hline
\end{tabular}

‡: Cell culture applications have not yet been documented for this device. 
Cassette layout

24 wells cassette $\bigcirc \square$ wells cassette
Liquids configuration

$\square$ Per row

Liquids library...

Liquid Feed
Reactor selection

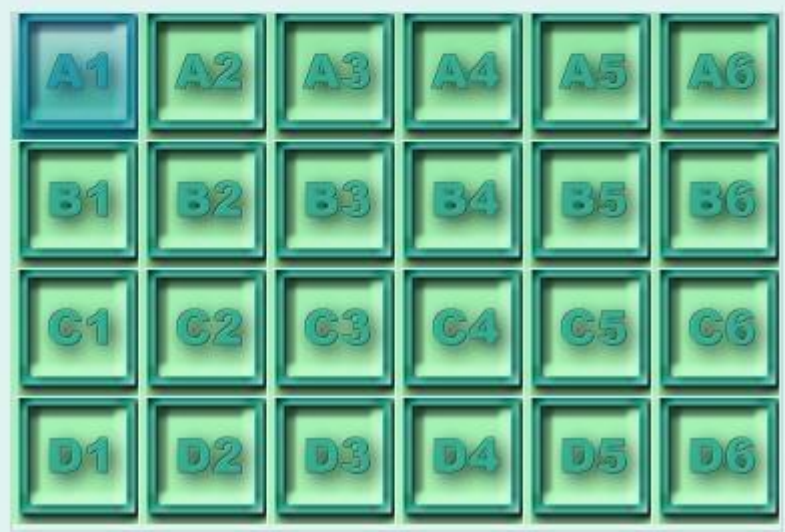

Control configuration

Library....

Configuration selection

Default: Cell

Reset PID integral buffer upon upload

Cut-off volume

Liquid

$4000 \mu l$

Actuator configuration

\begin{tabular}{|c|c|c|c|c|c|}
\hline & Level 1 & Level 2 & Level 3 & Level 4 & Level 5 \\
\hline$\triangle \mathrm{dO} 2$ & $\pm \quad 02$ & & & & \\
\hline $8 \mathrm{dO} 2$ & $\therefore \quad \mathrm{N} 2$ & & & & \\
\hline$\triangle \mathrm{pH}$ & \begin{tabular}{|l|l}
$\square$ & Gas D
\end{tabular} & & & & \\
\hline $8 \mathrm{pH}$ & $\approx \quad \mathrm{CO} 2$ & & & & \\
\hline$\Rightarrow$ Direct & (\$) Liquid & & & & \\
\hline
\end{tabular}

Reset to default Description

Figure 3: Actuator Setup in the micro-Matrix HMI 
a)

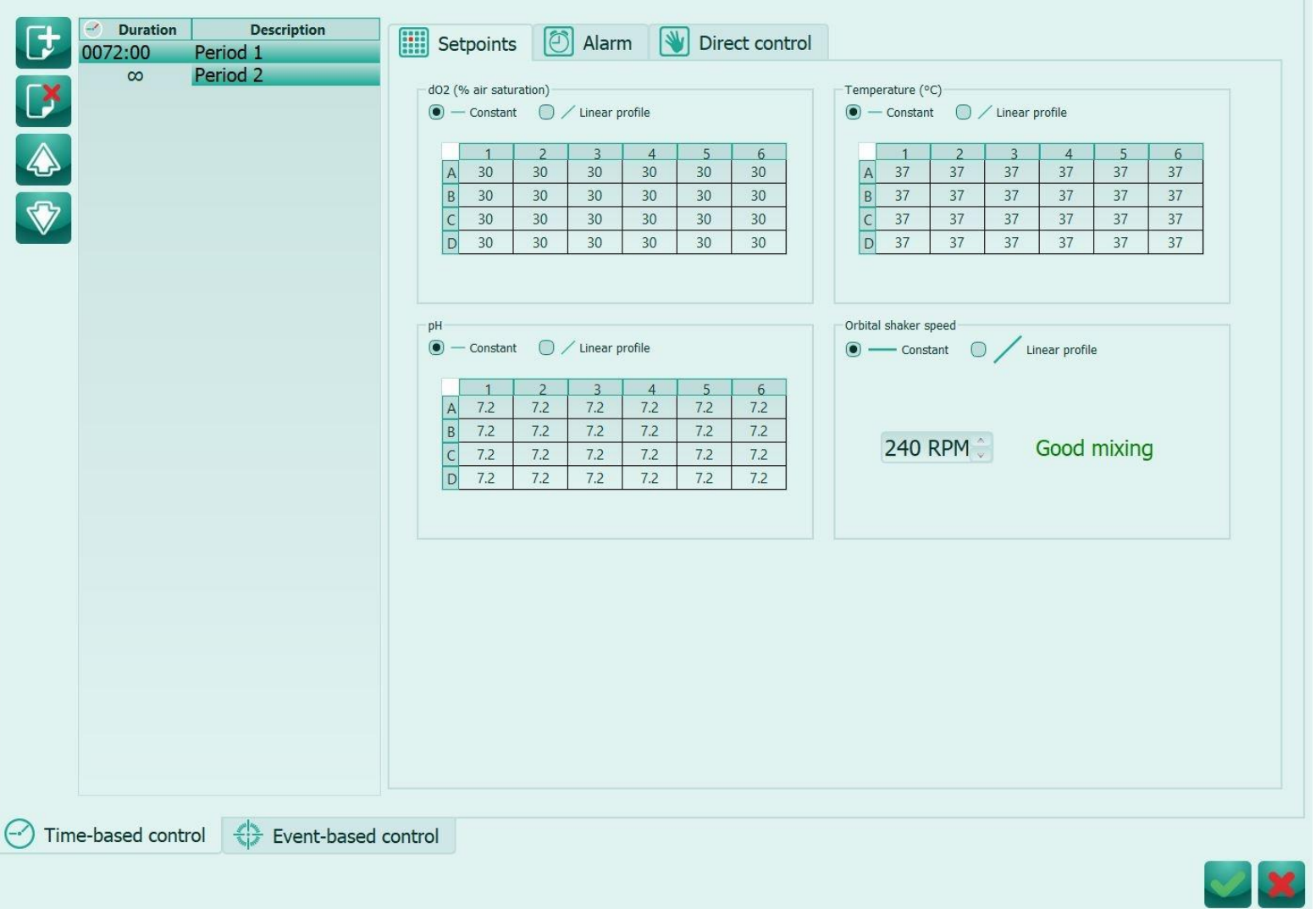

b)

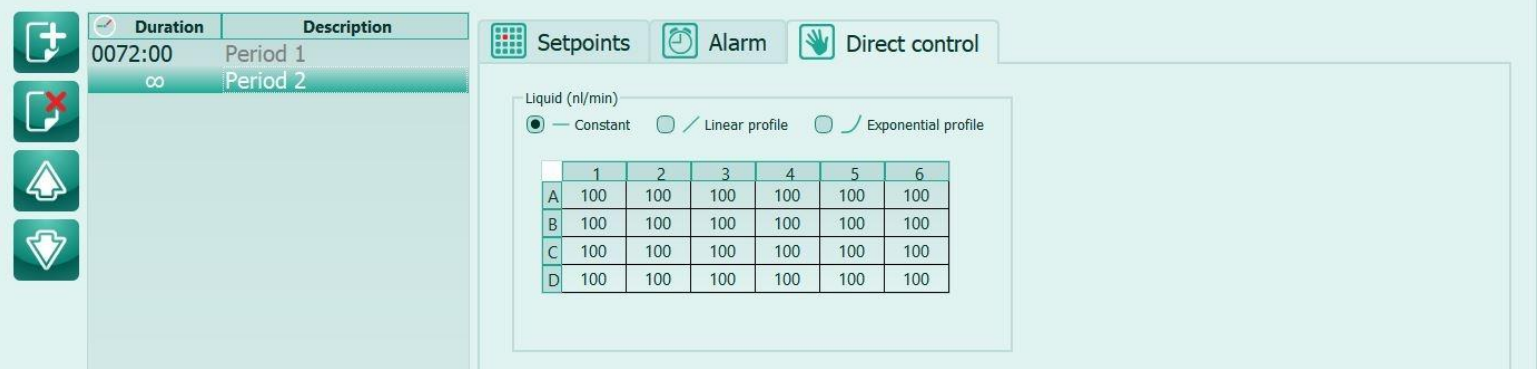

Figure 2: Setup of the experimental conditions in the micro-Matrix HMI. (a) Dissolved oxygen $\left(\mathrm{dO}_{2}\right)$, $\mathrm{pH}$, temperature and shaking speed. (b) Liquid additions 